\title{
Quantum Distribution-function Transport Equations in Non-normal Systems and in Ultra-fast Dynamics of Optically-excited Semiconductors
}

\author{
F. A. BUOT \\ Naval Research Laboratory, Washington, D.C. 20375-5320
}

\begin{abstract}
The derivation of the quantum distribution-function transport equations combines the Liouvillian super-Green's function technique and the lattice Weyl-Wigner formulation of the quantum theory of solids. A generating super-functional is constructed which allows an algebraic and straightforward application of quantum field-theoretical techniques in real time to derive coupled quantum-transport, condensate, and pairwavefunction equations. In optically-excited semiconductors, quantum distributionfunction transport equations are given for phonons, plasmons, photons, and electronhole pairs and excitons by transforming the Bethe-Salpeter equation into a multi-time evolution equation. The virtue of quantum distribution function is that it allows easy application of 'device-inflow' subsidiary boundary conditions for simulating femtosecond device-switching phenomena.
\end{abstract}

Keywords: Quantum distribution function, quantum transport, ultra-fast optics, excitons, nonequilibrium superconductivity, nonequilibrium superfluidity, lattice Weyl transform, Liouvillian, super Green's function

\section{INTRODUCTION}

There is a need for generalized quantum distribution-function transport equations, valid for nonnormal, non-uniform, and ultra-fast systems, as bases for large-scale computer simulations. This becomes urgent with advances in material science, ultra-fast laser probes, nanofabrication, and the development of more powerful energy beams. The drive to produce systems which are functionally more dense and have wider bandwidths will lead nanostructure devices to atomic-scale dimensions with different materials: insulators, semiconductors, metals, and superconductors.

The nonequilibrium quantum transport theory including pairing dynamics is formulated in terms of the Liouville-space (L-space) quantum-field theory [1-2] and lattice Weyl transform technique $[3,4]$. For normal systems, this reduces to the nonequilibrium Green's function technique of Schwinger [5], Kadanoff and Baym [6], and Keldysh [7], coupled with the lattice Weyl-Wigner formulation of the quantum theory of solids [3, 4]. Several new results are derived with the present approach. 
This L-space approach has provided the action principle for a multi-variable functional theory of nonequilibrium condensed-matter systems [8-10]. Thus, the method set forth here may open doors for the investigation of ultra-fast dynamics in quantum nanostructures. So far, only the distribution-function approach has characterized, in time-domain, a highly-nonlinear and highlynonequilibrium quantum behavior [11-14].

\section{QUANTUM DYNAMICS IN LIOUVILLE SPACE}

The density-matrix equation of quantum statistical dynamics in Hilbert space (H-space) becomes a super-Schrodinger equation for the super-state vector in L-space as

$$
\left.\left.i \hbar \frac{\partial}{\partial t}|\rho(t)\rangle\right\rangle=\mathscr{L}|\rho(t)\rangle\right\rangle
$$

$\rho(t)$ is the density-matrix operator for the whole many-body system in $\mathrm{H}$-space, and $|\rho(t)\rangle\rangle$ is the corresponding super-state vector in $\mathrm{L}$-space. The super-operator $\mathscr{L}$ corresponds to the commutator $[\mathscr{H}, \rho]$, and is referred to in this paper as the Liouvillian. Thus, we may write the Liouvillian $\mathscr{L}$ as $\mathscr{L}=\hat{\mathscr{H}}-\tilde{\mathscr{H}}$, which define $\hat{\mathscr{H}}$ and $\tilde{\mathscr{H}}$. These have the property that $\hat{\mathscr{H}}|\rho(t)\rangle\rangle=|\mathscr{H} \rho(t)\rangle\rangle$, and $\left.\tilde{\mathscr{H}}|\rho(t)\rangle\rangle=\left|\rho(t) \mathscr{H}^{\dagger}\right\rangle\right\rangle$. These relations are valid for fermions and bosons. For number-conserving fermion operator $\mathscr{H}, \tilde{\mathscr{H}}=\tilde{\mathscr{H}}^{\dagger}$. The quantum field super-operators, $\hat{\psi}(\tilde{\psi})$ and $\hat{\psi}^{\dagger}\left(\tilde{\psi}^{\dagger}\right)$, are defined through their commutation relations in H-Space.

\section{SUPER S-MATRIX THEORY IN L-SPACE}

The "transition probability" in L-space is given by the following relation

$$
\langle\langle\Lambda(t) \mid \rho(t)\rangle\rangle=\left\langle\left\langle 1|\bar{S}(\infty,-\infty)| \rho_{\mathrm{eq}}\right\rangle\right\rangle
$$

where $|1\rangle\rangle$ is a unit super-vector. We have

$$
\begin{aligned}
& \left.|\rho(t)\rangle\rangle=T \exp \left\{\frac{-i}{\hbar} \int_{0}^{t} \mathscr{L}_{0}\left(t^{\prime}\right) d t^{\prime}\right\} \bar{S}(t,-\infty)\left|\rho_{\mathrm{eq}}\right\rangle\right\rangle \\
& \left.|\Lambda(t)\rangle\rangle=T^{\mathrm{ac}} \exp \left\{\frac{-i}{\hbar} \int_{t}^{0} \mathscr{L}_{0}\left(t^{\prime}\right) d t^{\prime}\right\} \bar{S}(t, \infty)|\mathcal{O}\rangle\right\rangle
\end{aligned}
$$

where $T^{\text {ac }}$ denotes anti-chronological time ordering and

$$
\begin{aligned}
\bar{S}(t,-\infty)= & T \exp \left\{\frac{i}{\hbar} \int_{0}^{t} \mathscr{L}_{0}\left(t^{\prime}\right) d t^{\prime}\right\} \\
& T \exp \left\{\frac{-i}{\hbar} \int_{-\infty}^{t} \mathscr{L}\left(t^{\prime}\right) d t^{\prime}\right\} \\
& T \exp \left\{\frac{-i}{\hbar} \int_{0}^{t} \mathscr{L}_{0}\left(t^{\prime}\right) d t^{\prime}\right\}
\end{aligned}
$$

The "transition probability" obeys the equality

$$
\left\langle\left\langle 1|\bar{S}(\infty,-\infty)| \rho_{\mathrm{eq}}\right\rangle\right\rangle=\exp \frac{i}{\hbar} W
$$

where $W$ is identified as the effective action. It can be shown that

$$
W=\int_{-\infty}^{\infty}\left\langle\left\langle\Lambda(t)\left|i \hbar \frac{\partial}{\partial t}-\mathscr{L}\right| \rho(t)\right\rangle\right\rangle d t
$$

This relation forms the basis of a time-dependent functional theory of condensed matter discussed by Rajagopal and Buot in a series of papers [8-10].

\section{GENERALIZED QUANTUM DISTRIBUTION FUNCTIONS}

We introduce a 4-component second quantized quantum field super-operators given by

$$
\Psi(1)^{T}=\left(\begin{array}{llll}
\hat{\psi}(1) & \tilde{\psi}^{\dagger}(1) \quad \hat{\psi}^{\dagger}(1) \quad \tilde{\psi}(1)
\end{array}\right)
$$

This is a generalization of the multi-component quantum field operator first introduced by Nambu [15], and by De Dominicis and Martin [16]. In 
terms of the $\Psi$ fields, a system Liouvillian $\mathscr{L}$ can in general be expressed as

$$
\begin{array}{r}
\mathscr{L}=\sum_{N=1}^{M} v(1,2,3, \ldots, N) \Psi(1) \Psi(2) \Psi(3) \\
\ldots \Psi(N)+\mathscr{L}_{\text {ext }}
\end{array}
$$

where the Schwinger source term is given by $\mathscr{L}_{\text {ext }}=\hat{\mathscr{H}}_{\text {ext }}-\tilde{\mathscr{H}}_{e, \text { ext }}=u(1) \Psi(1)+u(12) \Psi(1) \Psi(2)$. The field super-operator averages can be written in terms of the $\bar{S}$-matrix, e.g.,

$$
\mathscr{G}(1,2)=\frac{(i \hbar)^{2} \frac{\delta^{2}\left\langle\left\langle 1|\bar{S}(\infty,-\infty)| \rho_{\mathrm{eq}}\right\rangle\right\rangle}{\delta u(2) \delta u(1)}}{\left\langle\left\langle 1|\bar{S}(\infty,-\infty)| \rho_{\mathrm{eq}}\right\rangle\right\rangle}
$$

We obtained the following ( $e=1$ for bosons, $e=-1$ for fermions),

$$
\mathscr{G}(1,2)=i \hbar\left(\begin{array}{cc}
F(1,2) & G(1,2) \\
e G^{T}(1,2) & { }^{\dagger} F(1,2)
\end{array}\right)
$$

where the superscript $T$ indicates the taking of the transpose, and,

$$
\begin{aligned}
F(1,2) & =\left(\begin{array}{ll}
g_{h h}^{c} & g_{h h}^{<} \\
g_{h h}^{>} & g_{h h}^{a c}
\end{array}\right)(1,2), \\
{ }^{\dagger} F(1,2) & =\left(\begin{array}{cc}
g_{e e}^{c} & e g_{e e}^{<} \\
e g_{e e}^{>} & g_{e e}^{a c}
\end{array}\right)(1,2) \\
G(1,2) & =\left(\begin{array}{cc}
G^{c} & e G^{<} \\
G^{>} & e G^{a c}
\end{array}\right)(1,2), \\
e G^{T}(1,2) & =\left(\begin{array}{cc}
e G^{c^{T}} & e G^{>^{T}} \\
G^{<^{T}} & G^{a c^{T}}
\end{array}\right)(1,2)
\end{aligned}
$$

$G(1,2)$ corresponds to the Keldysh nonequilibrium Green's function. We refer to the $\mathscr{G}(1,2)$ simply as moment quantum distribution function. Moments are defined for time-ordered quantum field superoperators. We also define quantum correlation functions or quantum cumulants, $\mathscr{K}$, analogous to the classical statistical theory. This distinction is important in treating the quantum transport of superfluids and quanta of real classical fields. We will also refer to both as generalized quantum distribution functions (GQDF).

In the application of Wick's theorem (for superfluid Bose system it is assumed that $\mathscr{L}_{0}$ is expanded about the condensate for Wick's theorem to be applicable) to evaluate the $\mathscr{G}$ 's in terms of diagrams or graphs, $\mathscr{K}(1,2, \ldots n)$ represents the topologically distinct 'connected' subset of graphs. We have

$$
\mathscr{K}(1,2, \ldots, n)=(i \hbar)^{n} \frac{\delta^{n} \ln \left\langle\left\langle 1|\bar{S}(\infty,-\infty)| \rho_{\mathrm{eq}}\right\rangle\right\rangle}{\delta u(n) \delta u(n-1) \ldots \ldots \delta u(1)}
$$

Similar functional derivative relations can be obtained between GQDF with even number of indices by using the variation with respect to the external Schwinger source term $u(1,2)$. Since $u(1,2)$ is an ordinary $c$-number, the order of the $u(i, j)$ 's is not critical in taking the functional derivatives.

\subsection{Self-Consistent Equations for Generalized Quantum Distribution Functions}

We have

$$
\left[\mathscr{G}^{0}(12)^{-1}-\breve{\Sigma}(12)\right]\left\{\frac{\mathscr{K}(23)}{i \hbar}\right\}=\delta(13)
$$

w h e r e $\mathscr{G}^{0}\left(\xi^{\prime \prime}, 2\right)^{-1}=\left(\tau_{4}^{e}\right)^{-1} i \hbar \delta\left(\xi^{\prime \prime}, 2\right) \partial / \partial t_{2}-$ $\mathscr{V}\left(\xi^{\prime \prime}, 2\right)$, where the $\left(\tau_{4}^{e}\right)$-matrix arise from the commutation relation of the $\Psi(i, j)$ 's, $\mathscr{V}\left(\xi^{\prime \prime}, 2\right)$ is a one-body potential matrix, and $\breve{\Sigma}(12)$ is the particle super self-energy matrix. For fermions, $\mathscr{G}(1,2) \equiv \mathscr{K}(1,2) . \breve{\Sigma}(12)$ is expressed in terms of $\mathscr{K}$ 's (up to second-order cumulants) and vertex functions. These vertex functions obey equations similar to the Dyson equation, involving functional derivative of the self-energy with respect to secondorder GQDF hence decoupling the BBKGY hierarchy. The self-energy due to e-e interaction includes the electron-plasmon vertex function.

\section{QUANTUM TRANSPORT EQUATIONS}

The time-evolution equation for $\mathscr{K}(i, j)$ is obtained. We write the resulting equations for 
the $2 \times 2$ matrix elements of $\mathscr{K}(i, j)$ as

$$
\begin{aligned}
& G^{0^{-1}} \tilde{G}=\delta+\Sigma \tilde{G}+\Delta^{\dagger} \mathfrak{J} \\
& G^{0^{-1^{T}}} \tilde{G}^{T}=\delta+\Sigma^{T} \tilde{G}^{T}+{ }^{\dagger} \Delta \mathfrak{I} \\
& G^{0^{-1^{T}} \dagger} \mathfrak{I}=\Sigma^{T \dagger} \mathfrak{I}+e^{\dagger} \Delta \tilde{G} \\
& G^{0^{-1}} \mathfrak{I}=e \Delta \tilde{G}^{T}+\Sigma \mathfrak{I}
\end{aligned}
$$

where $\Delta$ is the pair potential or gap function, and $G^{0^{-1}}$ is a diagonal matrix with elements proportional to $i \hbar \delta(12) \partial / \partial t_{2}-\bar{v}(12)$ with $\bar{v}(12)$ proportional to a one-body external potential. These equations were also given by Aronov, et al. [17a] and formally resemble the well-known Gorkov equations [17b] for superconductors at thermal equilibrium.

Solving quantum transport problems [11] centers on the evolution of $\rho^{<}(12) \equiv e i \hbar G^{<}(12)$, which happens to be one of the matrix elements of the nonequilibrium matrix Green's function of Eq. (9). $\tilde{G}^{>,<}$contains all information about the statistical aspects of the field intensity. This is coupled to the 'advanced' and 'retarded' propagators often directly related to the experiment and contain all the energetics and dynamical information of the system. We obtain the transport equation for $\tilde{G}^{>,<}$given by the following expressions.

\subsection{Nonequilibrium Superconductivity}

We have the following expressions for superconductors,

$$
\begin{aligned}
i \hbar\left(\frac{\partial}{\partial t_{1}}\right. & \left.+\frac{\partial}{\partial t_{2}}\right) G^{>,<}(12)=\left[\bar{v}+\operatorname{Re} \Sigma^{r}, G^{>,<}\right] \\
& -\left[\operatorname{Re} G^{r}, \Sigma^{>,<}\right] \\
& -\frac{i}{2}\left\{\Gamma, G^{>,<}\right\}+\frac{i}{2}\left\{A, \Sigma^{>,<}\right\} \\
& +\left\{\Delta_{h h}^{r} g_{e e}^{>,<}+g_{h h}^{>,<} \Delta_{e e}^{r}\right\} \\
& +\left\{\Delta_{h h}^{>,<} g_{e e}^{a}+g_{h h}^{a} \Delta_{e e}^{>,<}\right\}
\end{aligned}
$$

$$
\begin{aligned}
& i \hbar\left(\frac{\partial}{\partial t_{1}}+\frac{\partial}{\partial t_{2}}\right) G^{r}(12)=\left[\left(\bar{v}+\Sigma^{r}\right), G^{r}\right] \\
& +\left[\Delta_{h h}^{r} g_{e e}^{r}-g_{h h}^{r} \Delta_{e e}^{r}\right]+\left\{\Delta_{h h}^{<} g_{e e}^{r}+g_{h h}^{r} \Delta_{e e}^{>}\right\} \\
& -\left\{\Delta_{h h}^{<} g_{e e}^{r}+g_{h h}^{r} \Delta_{e e}^{<}\right\}+\left[g_{h h}^{>}-g_{h h}^{<}\right] \Delta_{e e}^{r}-\left[g_{h h}^{>}\right. \\
& \left.-g_{h h}^{<}\right]\left[\Delta_{e e}^{>}-\Delta_{e e}^{<}\right]
\end{aligned}
$$

Equations for the pair wavefunctions, $g_{e e}^{>,<}, g_{e e}^{r}$, $g_{h h}^{><}, g_{h h}^{r}$, etc, are also obtained. Using lattice Weyl transformation, we can transform the above total time evolution equations, Eqs. (17) - (18) into quantum transport equations in $(\mathbf{p}, \mathbf{q}, E, t)$ phase space for superconductive. systems.

\subsection{Nonequilibrium Bose Superfluids}

The expressions for $i \hbar\left(\partial / \partial t_{1}+\partial / \partial t_{2}\right) \tilde{G}^{>,<}(12)$, $i \hbar\left(\partial / \partial t_{1}+\partial / \partial t_{2}\right) \tilde{G}^{r}(12)$, and pair-wavefunction equations, are similarly obtained by taking $e=1$ in Eqs. (13) - (16) and applying the self-consistency condition for the boson-particle self-energies. We can also transform the total time evolution equations into quantum transport equations in $(\mathbf{p}, \mathbf{q}, E, t)$ phase space for Bose superfluid systems [18]. This will not be given in this paper.

\section{ULTRA-FAST DYNAMICS OF EXCITED SEMICONDUCTORS}

The physics of highly-excited semiconductor heterostructures has been of continuing research interest [19-23]. The electron-hole $(\mathrm{e}-\mathrm{h})$ pair theory is formally identical to the theory of superconductivity for extremely high-density of $\mathrm{e}-\mathrm{h}$ pairs. However, in the low density limit real electron-hole pair bound states do occur, these are localized composite $\mathrm{e}-\mathrm{h}$ elementary excitations called 'excitons' [23]. The device physics [24] of interacting matter and radiation requires the consideration of all ranges of $\mathrm{e}-\mathrm{h}$ densities and short-time dynamics.

The multi-component (in the "hat" and "tilde" indices) quantum field super-operators for electro- 
magnetic field (transverse and longitudinal) and lattice vibrations are given, respectively, by

$$
\begin{gathered}
A(\zeta)=\frac{1}{\sqrt{4 \pi c}}\left(\begin{array}{c}
\hat{A}(\zeta) \\
\tilde{A}(\zeta)
\end{array}\right), \Theta(\zeta)=\left(\begin{array}{c}
\hat{\vartheta}(\zeta) \\
\tilde{\vartheta}(\zeta)
\end{array}\right) \\
\text { and } U(\xi)=\left(\begin{array}{c}
\sqrt{m_{k}} \hat{u}(\xi) \\
\sqrt{m_{k}} \tilde{u}(\xi)
\end{array}\right)
\end{gathered}
$$

We used a "composite field operator" as the fourth field, $\Psi(1,2)$, since coupling to fermions only occur through bilinear product of fermion fields. This is made up of $\mathscr{F}(12), \Re(12), e \Re^{T}(12)$, and ${ }^{\dagger} \mathscr{F}(12)$, consisting of different combination of the "hat" and "tilde" indices. We have,

$\Psi(1,2)^{T}=\left(\mathscr{F}(1,2) \Re(1,2) \quad e \Re^{T}(1,2) \quad \dagger \mathscr{F}(1,2)\right)$

The time-ordered averages of the components give the familiar quantities, namely,

$$
\langle T \Psi(1,2)\rangle=\mathscr{K}(1,2)=\left(\begin{array}{cc}
F(1,2) & G(1,2) \\
e G^{T}(1,2) & { }^{\dagger} F(1,2)
\end{array}\right)
$$

The scalar potential field super-operator $\Theta(\zeta)$ is not an independent field [25].

\section{ELECTRON-HOLE AND EXCITON TIME EVOLUTION EQUATION}

The super propagator for electron-hole and exciton is defined by

$$
\mathscr{K}(\overline{2} \overline{3} ; 12)=\frac{\delta \mathscr{K}(\overline{2} \overline{3})}{\delta f^{\operatorname{ext}}(12)}
$$

$f^{\text {ext }}(12)$ includes the matrix element of the dipole moment containing the selfconsistent transverse electromagnetic field in the microcavity, for example. In the matrix definition of $\mathscr{K}(12 ; 34)$, the nonequilibrium pair propagator is identified as one of the matrix elements, namely, the ' $(1,4 \mathrm{th})$ 'component.

\subsection{Schrödinger - Wannier Equation for the Exciton Wavefunction}

It is helpful to derive from the Bethe-Salpeter equation, the Schrödinger-Wannier equation for the electron-hole pair wavefunction. Neglecting the self-energy terms and retaining only the Coulomb interaction terms, we obtain after setting $t_{1}=t_{2}=t$

$$
\begin{aligned}
\frac{i}{\hbar} \frac{\partial}{\partial t} \varphi(12) & =\left[-\left\{\frac{\hbar^{2}}{2 m_{1}} \nabla_{1}^{2}+\frac{\hbar^{2}}{2 m_{2}} \nabla_{2}^{2}\right\}\right. \\
& \left.-\frac{e^{2}}{\left|r_{1}-r_{2}\right|}\right] \varphi(12)
\end{aligned}
$$

This is the familiar Schrödinger equation for a two-particle system consisting of an electron and a hole. Using a dielectric function to screen the potential, the resulting bound states correspond to exciton states [23].

\subsection{Time Evolution Equations for $\mathscr{K}(12 ; 34)$}

The total time derivative of $\mathscr{K}(12 ; 34)$ is obtained from the Bethe-Salpeter equation, the details will be published elsewhere. The ' $(1,4$ th)' element describing the electron-hole pair propagator is denoted by $\mathscr{G}_{\text {exciton }}(12 ; 34)$, which in turn contains in its ' $(1,4)$ th' element the $\mathrm{e}-\mathrm{h}$ pair density matrix $\mathscr{G}_{\text {exciton }}^{<}(12 ; 34)$.

\subsection{Transport Equation for the Nonequilibrium Pair Propagator}

What needs to be done is to single out the equation for the electron-hole pair density matrix, which we denote by $\mathscr{G}_{\text {exciton }}^{<}(12 ; 34)$. The result is very long and will be published elsewhere. Interested readers are encouraged to contact the authors for this detail. The final transport equation is obtained by setting $t_{1}=t_{2}$, and $t_{3}=t_{4}$ and taking the lattice Weyl transform of the above transport equation [in general double lattice Weyl transform in spatial variables]. To demonstrate this, let us take the simplest approximation of this equation, which we 
write us

$$
\begin{aligned}
i \hbar( & \left.\frac{\partial}{\partial t_{1}}+\frac{\partial}{\partial t_{3}}\right) \mathscr{G}_{\text {exciton }}^{<}\left(12, t_{1} ; 34, t_{3}\right) \\
\quad= & \left\{\begin{array}{c}
{\left[\overline{\mathrm{v}}\left(12^{\prime \prime}\right)\right] \mathscr{G}_{\text {exciton }}^{<}\left(2^{\prime \prime} 2, t_{1} ; 34, t_{3}\right)} \\
-\mathscr{G}_{\text {exciton }}^{<}\left(12^{\prime \prime}, t_{1} ; 34, t_{3}\right)\left[\overline{\mathrm{v}}^{T}\left(2^{\prime \prime} 2\right)\right]
\end{array}\right\} \\
& -\left\{\begin{array}{c}
{\left[\overline{\mathrm{v}}\left(33^{\prime \prime}\right)\right] \mathscr{G}_{\text {exciton }}^{<}\left(12, t_{1} ; 3^{\prime \prime} 4, t_{3}\right)} \\
-\mathscr{G}_{\text {exciton }}^{<}\left(12, t_{1} ; 34^{\prime \prime}, t_{3}\right)\left[\overline{\mathrm{v}}^{T}\left(4^{\prime \prime} 4\right)\right]
\end{array}\right\}
\end{aligned}
$$

Upon taking the lattice Weyl transform and taking the gradient expansion the result is

$$
\begin{aligned}
& i \hbar \frac{\partial}{\partial t} \mathscr{G}_{\text {exciton }}^{<}\left(p_{1}, q_{1} ; p_{2}, q_{2} ; E, t\right) \\
&=-i \hbar\left\{\nabla_{p_{1}} \in\left(p_{1}\right) \bullet \nabla_{q_{1}}\right. \\
&\left.\mathscr{G}_{\text {exciton }}^{<}\left(p_{1}, q_{1} ; p_{2}, q_{2} ; E, t\right)\right\} \\
&+ i \hbar\left\{\nabla_{p_{2}} \in\left(p_{2}\right) \bullet \nabla_{q_{2}}\right. \\
&\left.\mathscr{G}_{\text {exciton }}^{<}\left(p_{1}, q_{1} ; p_{2}, q_{2} ; E, t\right)\right\} \\
& \frac{1}{\left(h^{3}\right)} \int d p_{1}^{\prime} K_{\bar{v}}^{s}\left(p_{1}, q_{1} ; p_{1}^{\prime}, q_{1}\right) \\
& \mathscr{G}_{\text {exciton }}^{<}\left(p_{1}^{\prime}, q_{1} ; p_{2}, q_{2} ; E, t\right) \\
&-\frac{1}{\left(h^{3}\right)} \int d p_{2}^{\prime} K_{\bar{v}}^{s}\left(p_{2}, q_{2} ; p_{2}^{\prime}, q_{2}\right) \\
& \mathscr{G}_{\text {exciton }}^{<}\left(p_{1}, q_{1} ; p_{2}^{\prime}, q_{2} ; E, t\right)
\end{aligned}
$$

The last equation is the appropriate Wigner distribution transport equation of a two-particle distribution corresponding to the SchrödingerWannier equation of Eq. (23)

\section{TRANSPORT EQUATIONS FOR PHONONS}

The "reduced density matrix" for phonons is defined by

$$
S_{i j}^{<}\left(\xi \xi^{\prime} ; t t^{\prime}\right)=\frac{i}{\hbar}\left\langle T \hat{u}_{i}(\xi, t) \tilde{u}_{j}\left(\xi^{\prime}, t^{\prime}\right)\right\rangle
$$

The lattice Weyl transform can be cast in the form

$$
\begin{aligned}
& -i S_{i j}^{<}\left(k, k^{\prime} ; p^{\prime \prime}, l_{c} ; \omega, t_{c}\right) \\
& =A_{i j}\left(k, k^{\prime} ; p^{\prime \prime}, l_{c} ; \omega, t_{c}\right) n\left(p^{\prime \prime}, l_{c} ; \omega, t_{c}\right)
\end{aligned}
$$

Similarly, we can write the phonon "hole" distribution as

$$
\begin{aligned}
& -i S_{i j}^{>}\left(k, k^{\prime} ; p^{\prime \prime}, l_{c} ; \omega, t_{c}\right) \\
& =A_{i j}\left(k, k^{\prime} ; p^{\prime \prime}, l_{c} ; \omega, t_{c}\right)\left[1+n\left(p^{\prime \prime}, l_{c} ; \omega, t_{c}\right)\right]
\end{aligned}
$$

\subsection{Phonon Transport Equations}

We obtain the following expressions

$$
\begin{aligned}
& \left(\frac{\partial^{2}}{\partial t^{2}}-\frac{\partial^{2}}{\partial t^{\prime 2}}\right) S^{>,<} \\
& =\left[\operatorname{Re} \Pi^{r}-d^{(2)}, S^{>,<}\right]+\left[\Pi^{>,<}, \operatorname{Re} S^{r}\right] \\
& +i\left\{\operatorname{Im} \Pi^{r}, S^{>,<}\right\}-i\left\{\Pi^{>,<}, \operatorname{Im} S^{r}\right\} \\
& =\left[\operatorname{Re} \Pi^{r}-d^{(2)}, S^{>,<}\right]+\left[\Pi^{>,<}, \operatorname{Re} S^{r}\right] \\
& \pm \frac{1}{2}\left\{\Pi^{>,<}, S^{<,>}\right\} \mp \frac{1}{2}\left\{\Pi^{<,>}, S^{>,<}\right\} \\
& \left(\frac{\partial^{2}}{\partial t^{2}}-\frac{\partial^{2}}{\partial t^{\prime 2}}\right) S^{r \cdot a}=-\left[d^{(2)}, S^{r . a}\right]+\left[\Pi^{r, a}, S^{r, a}\right]
\end{aligned}
$$

where we have used the definition for the force constants

$$
d_{i j}^{(2)}\left(\xi \xi^{\prime}\right)=\frac{\phi_{i j}^{(2)}\left(\xi \xi^{\prime}\right)}{\sqrt{m_{k} m_{k^{\prime}}}}
$$

and the following identities

$$
i \operatorname{Im} S^{r}=\frac{S^{>}-S^{<}}{2}, \quad i \operatorname{Im} \Pi^{r}=\frac{\Pi^{>}-\Pi^{<}}{2}
$$

What then take the lattice Weyl transform of Eqs. (29) and (30) to obtain the transport equations in $\left(\vec{p}, \vec{X}_{l_{c}}, \omega, t\right) \equiv(\vec{p}, \vec{q}, E, t)$ phase-space. 


\subsection{The Phonon Boltzmann Equation}

More revealing equations can be seen by neglecting off-diagonal or "inter branch" terms, and expanding the equations in terms of the gradients. We defined a renormalized kinematic frequency by $\Omega_{\lambda \lambda}^{2}(p, q, E, t)=\omega_{\lambda \lambda}^{o}{ }^{2}-\operatorname{Re} \prod_{\lambda \lambda}^{r}(p, q, E, t)$. The Boltzmann equation for the distribution function of phonons, $n_{\lambda \lambda}(p, q, E, t)$, from vibration branch $\lambda$ readily follows by neglecting leading quantum corrections. We obtain the familiar interpretation [22] relating $(i / 2)\left\{\hbar \Pi_{\lambda \lambda}^{<}(p, q, E, t) / E\right\}$ as the scattering-out rate and $(i / 2)\left\{\hbar \Pi_{\lambda \lambda}^{<}(p, q, E, t) / E\right\}$ as the scattering-in rate. By taking the renormalized frequency to be given by the solution of the equality $\Omega_{\lambda \lambda}(p, q, \omega t)=\omega$, with this solution denoted by $\omega_{\lambda}(p, t)$, we finally obtain

$$
\begin{gathered}
\frac{\partial}{\partial t} n_{\lambda}\left(p, q, \omega_{\lambda}, t\right)+\nabla_{p} \hbar \omega_{\lambda}(p, t) \bullet \nabla_{q} n_{\lambda}\left(p, q, \omega_{\lambda}, t\right) \\
=-\frac{i \prod_{\lambda}^{>}\left(p, q, \omega_{\lambda}, t\right)}{2 \omega_{\lambda}(p)} n_{\lambda}\left(p, q, \omega_{\lambda}, t\right) \\
+\frac{i \prod_{\lambda}^{<}\left(p, q, \omega_{\lambda}, t\right)}{2 \omega_{\lambda}(p)}\left[1+n_{\lambda}\left(p, q, \omega_{\lambda}, t\right)\right]
\end{gathered}
$$

The renormalized group velocity [26] emerges since phonons do not diffuse freely but interact with the environment as well as collide with each other. Similar situation arises in deriving the plasmon and photon Boltzmann equations. We expect the leading term of $\operatorname{Re} \prod_{\lambda \lambda}^{r}(p, q, E, t)$ to be independent of time. An example of the RHS of Eq. (33) for phonon-phonon interaction can be found in the work of the author [27] and in the study of phonon hydrodynamics and second sound [28].

\section{TRANSPORT EQUATIONS FOR PLASMONS}

\subsection{The Plasmon Boltzmann Equation}

The plasmon super-propagator is given by $D_{o}\left(\zeta, \zeta^{\prime}\right)=\delta\langle\Theta(\zeta)\rangle / \delta \rho^{\mathrm{ext}}\left(\zeta^{\prime}\right)$. The plasmon Boltz- mann equation is obtained by means of gradient expansion. We have

$$
\begin{aligned}
\frac{\partial}{\partial t} D_{o}^{>,}<(p, q, E, t)+\left[\frac{\frac{2}{\hbar^{2}} p-\nabla_{p} \operatorname{Re} \prod_{o}^{r}(p, q, E, t)}{\frac{\partial}{\partial E} \operatorname{Re} \prod_{o}^{r}(p, q, E, t)}\right] \\
\bullet \nabla_{q} D_{0}^{<,>}(p, q, E, t) \\
\quad=\mp \frac{i}{2}\left\{\frac{\prod_{o}^{>,<}(p, q, E, t)}{\hbar \frac{\partial}{\partial E} \operatorname{Re} \prod_{o}^{r}(p, q, E, t)} D_{0}^{<,>}(p, q, E, t)\right\} \\
\quad \pm \frac{i}{2}\left\{\frac{\prod_{o}^{<,>}(p, q, E, t)}{\hbar \frac{\partial}{\partial E} \operatorname{Re} \prod_{o}^{r}(p, q, E, t)} D_{o}^{>,<}(p, q, E, t)\right\}
\end{aligned}
$$

where we have left out terms involving diffusion in momentum and energy space. We would like to point out the notable use of the renormalized plasmon group velocity given by $\left(\frac{2}{\hbar^{2}} p-\nabla_{p} \operatorname{Re} \prod_{o}^{r}(p, q, E, t)\right) / \frac{\partial}{\partial E} \operatorname{Re} \prod_{o}^{r}(p, q, E, t)$. The use of the renormalized diffusion velocity arises from the same reason as that given in the derivation of the Boltzmann equation for phonons. The right-hand side are the familiar collision terms. The omitted terms describe the kinematics of the dynamical motion of plasmons.

\section{TRANSPORT EQUATIONS FOR PHOTONS}

We will also neglect the off-diagonal terms in polarization indices. The result to leading order is given by

$$
\begin{aligned}
& \frac{\partial}{\partial t} F_{\lambda \lambda}^{>,<}(p, q, E, t)+\frac{\Omega_{\lambda \lambda}(p, q, E, t)}{\omega} \\
& \nabla_{p} \hbar \Omega_{\lambda \lambda}(p, q, E, t) \bullet \nabla_{q} F_{\lambda \lambda}^{>,<}(p, q, E, t) \\
& =-\frac{i}{2}\left\{\frac{P_{\lambda \lambda}^{>}(p, q, E, t)}{\omega}\right\} F_{\lambda \lambda}^{<}(p, q, E, t) \\
& +\frac{i}{2}\left\{\frac{P_{\lambda \lambda}^{<}(p, q, E, t)}{\omega}\right\} F_{\lambda \lambda}^{>}(p, q, E, t)
\end{aligned}
$$

where $\Omega_{\lambda \lambda}^{2}(p, q, E, t)=c^{2} p^{2}-\operatorname{Re} P_{\lambda \lambda}^{r}(p, q, E, t)$ and we have neglected the leading quantum corrections on the right-hand side. We note that $P^{r}$ is related to the transverse dielectric tensor [29]. We may 
consider $\Omega_{\lambda \lambda}^{2}(p, q, E, t)>0$ to constitute the validity of the Boltzmann equation for photons with diffusive term. The use of renormalized group velocity corresponds to the use of refractive index well-known in optics.

A self-consistent Boltzmann transport equation follows by setting, $\Omega_{\lambda \lambda}^{2}(p, q, \omega, t)=c^{2} p^{2}-$ $\operatorname{Re} P^{r}(p, q, \omega, t)=\omega^{2}>0$. Upon substituting the solution for $\omega$, which we will denote by $\omega_{\lambda}(p, t)$, we obtain

$$
\begin{aligned}
\frac{\partial}{\partial t} F_{\lambda \lambda}^{>,<} & \left(p, q, \omega_{\lambda}, t\right)+\nabla_{p} \hbar \omega_{\lambda}(p, t) \bullet \nabla_{q} F_{\lambda \lambda}^{>,<}\left(p, q, \omega_{\lambda}, t\right) \\
& =-\frac{i}{2}\left\{\frac{P_{\lambda \lambda}^{>}\left(p, q, \omega_{\lambda}, t\right)}{\omega_{\lambda}}\right\} F_{\lambda \lambda}^{<}(p, q, E, t) \\
& +\frac{i}{2}\left\{\frac{P_{\lambda \lambda}^{<}\left(p, q, \omega_{\lambda}, t\right)}{\omega_{\lambda}}\right\} F_{\lambda \lambda}^{>}(p, q, E, t)
\end{aligned}
$$

\section{CONCLUDING REMARKS}

A major challenge in analyzing the ultra-fast dynamics of semiconductor gain material for investigating microcavity lasers is the fact that the electron-hole system properties are strongly affected by many-particle Coulomb interactions, and strong coupling to the light and crystal latticephonon fields [24]. In device physics of highly excited semiconductor systems, involving all ranges of $\mathrm{e}-\mathrm{h}$ pair densities, the exciton, $\mathrm{e}-\mathrm{h}$ Cooper pairs, and $\mathrm{e}-\mathrm{h}$ plasma energetics and their accompanying distributions greatly affect the polaritons, phonons, biexcitons, and higher-order 'pairing' (excitonic molecules) distributions, their wavefunctions and energies. For the bosons of real fields, the diffusion velocity is not equal to the group velocity of bare excitations but is defined only by its renormalized value. Quanta of these classical fields interact with the environment or collide with each other as it diffuse in space. The condensate and normal excitation energetics and their accompanying distribution also influence the gap function and energy gap. The full transport equations will be published elsewhere.

\section{Acknowledgement}

The author is grateful to Dr. A. K. Rajagopal for helpful discussions. This work is supported in part by the Office of Naval Research.

\section{References}

[1] Schmutz, M. (1978). "Real-time Green's functions in many-body problems", Z. Physik, B30, 97-106; Buot, F. A. and Rajagopal, A. K. (1994). "Quantum transport using Liouvillian quantum-field dynamics and functional approach to self-consistent many-body and scattering effects", in Proc. Third Int. Workshop on Comp. Electronics, Corvallis: Oregon State University, pp. 183-186.

[2] Arimatsu, T. and Umezawa, H. (1987). "General structure of non-equilibrium thermo field dynamics", Prog. Theor. Phys., 77, 53-66; Suzuki, M. (1985). "Thermo field dynamics in equilibrium and non-equilibrium quantum systems", J. Phys. Soc. Jpn., 54, 44834485.

[3] Buot, F. A. (1974). "Method for calculating $\operatorname{Tr} \mathscr{H}^{n}$ in solid state theory", Phys. Rev., B10, 3700-3705; "Weyl transform and the magnetic susceptibility of relativistic Dirac electron gas", Phys. Rev., A8, 1570-1581, 1973 \& Phys. Rev, A9, 211, 1974; "Formalism of distributionfunction method in impurity screening", Phys. Rev, B14, 977-989, 1976; "Magnetic susceptibility of interacting free and Bloch electrons", Phys. Rev., B14, 3310-3328, 1976; "Real-space tight-binding and discrete phase-space many-body quantum transport", Superlattices and Microstructures 11, 103-111, 1992. See also P. Kasperkovitz,"Wigner-Weyl formalisms for toroidal geometries", Ann. Phys., 230, 21, 1994 (Note that for crystals with inversion symmetry, there is an odd number of lattice points obeying the Born-von Karman periodic boundary condition).

[4] Buot, F. A. and Jensen, K. L. (1990). "Lattice WeylWigner formulation of exact many-body quantum transport theory and applications to novel quantum-based devices", Phys. Rev., B42, 9429-9457; Buot, F. A. "Exact integral operator form of the Wigner distribution function equation in many-body quantum transport theory", J. Stat. Phys., 61, 1223-1256, 1990.

[5] Schwinger, J. (1961). "Brownian motion of a quantum oscillator", J. Math Phys., 2, 407-432.

[6] Kadanoff, L. P. and Baym, G. (1962). Quantum statistical Mechanics. New York: Benjamin.

[7] Keldysh, L. V. (1964). "Diagram technique for nonequilibrium processes", Zh. Eksp. Theor. Phys., 47, 1515, [1965, Sov. Phys.-JETP 20, 1018]. See also V. Korenman, "Nonequilibrium quantum statistics: application to lasers", Annals Phys., 39, 72-126, 1966; P. Danielewicz, "Quantum theory of nonequlibrium processes, I", Annals Phys., 152, 239-304, 1995.

[8] Rajagopal, A. K. and Buot, F. A. (1965). "A nonequilibrium time-dependent functional theory based on Liouvillian quantum field dynamics", Int. J. Quantum Chem., 56, 389-397.

[9] Rajagopal, A. K. and Buot, F. A. (1995). "Nonequilibrium time-dependent functional theory for coupled 
interacting fields", Phys. Rev., B51, 1883-1897; "Timedependent functional theory of superconductors", Phys. Rev., B52, 6769-6774, 1995.

[10] Rajagopal, A. E. and Buot, F. A. (1996). "Generalized functional theory of interacting coupled Liouvillian quantum fields of condensed matter", Topics in Current Chemistry 181 Density functional theory II. New York: Springer Verlag.

[11] Buot, F. A. (1993). "Mesoscopic physics and nanoelectronics: nanoscience and nanotechnology", Phys. Reports, 234, 73-174.

[12] Jensen, K. L. and Buot, F. A. (1991). "Numerical simulation of intrinsic bistability and high-frequency current oscillations in resonant tunneling structures", Phys. Rev. Lett., 66, 1078-1081; Biegel B. A. and Plummer, J. D. "Comparison of self-consistency iteration options for the Wigner function method of quantum device simulation", Phys. Rev. B, 54, 80708082, 1996.

[13] Buot, F. A. and Jensen, K. L. (1991). "Intrinsic highfrequency oscillations and equivalent circuit model in the negative differential resistance region of resonant tunneling devices", COMPEL, 10, 241.

[14] Buot, F. A. and Rajagopal, A. K. (1993). "Highfrequency behavior of quantum-based devices: equivalent circuit, nonperturbative response, and phase-space analyses", Phys. Rev., B48, 17217-17232. See also, "Theory of novel nonlinear quantum transport effects in resonant tunneling structures", Materials Sc. and Engng., B35, $303-317,1995$.

[15] Nambu, Y. "Quasi-particles and gauge invariance in the theory of superconductivity", Phys. Rev., 117, 648-663, 1960 ; see also P.W. Anderson, "Random-phase approximation in the theory of superconductivity", Phys. Rev., 112,1900, 1958 ; A. K. Rajagopal, "Spin waves in an interacting electron gas", Phys. Rev., 142, 152, 1966.

[16] De Dominicis, C. and Martin, P. C. (1964). "Stationary entropy principle and renormalization in normal and superfluid systems. I. Algebraic formulation", J. Math. Phys., 5, 14-30.

[17a] Aronov, A. G., Gal'perin, Yu M., Gurevich, V. L. and Kozub, V. I. in Langenberg, D. N. and Lakin, A. I. "Kinetic approach", Eds., Nonequilibrium Superconductivity, New York: North Holland, 1986, pp. 325-376.

[17b] For a discussion on Gorkov equations, see for example A. L. Fetter and J. D. Walecka, Quantum Theory of Many-Particle Systems, New York: McGraw Hill, 1971, p. 183.

[18] Kirkpatrick, T. R. and Dorfman, J. R. (1985). "Transport in dilute but condensed nonideal Bose gas: kinetic equations", J. Low Temp. Phys., 58, 301-331.

[19] Yokoyama, H. (1992). "Physics and device applications of optical microcavities", Science, 256, 66.

[20] Keldysh, L. V. (1992). "Coherent excitonic molecules", Solid St. Comm., 84, 37-43; L. V. Keldysh, "Macroscopic coherent states of excitons in semiconductors", in Bose-Einstein Condensation, eds. A. Griffin, D.W. Stoke, and S. Stringari , Cambridge: Univ. Press, 1995

[21] Schmitt-Rink, S., Chemla, D. S. and Haug, H., (1988). "Nonequilibrium theory of the optical Stark effect and spectral hole burning in semiconductors", Phys. Rev., B37, 941 .

[22] Koinov, Z. G. and Glinskii, G. F. (1988). "A new approach to the theory of polaritons in semiconductors at finite temperatures: local-field effects and crystal optics approximation", J. Phys. A: Math. Gen., 21, 3431-3450; see also Z. G. Koinov (1990). "Self-consistent approach to the theory of Wannier excitons in polar semiconductors", J. Phys. Condens. Matter., 2, 6507-6518.

[23] Knox, R. (1963). "Excitons", Solid State Phys. Suppl., 5, 2.

[24] Jahnke, F. and Koch, S. W. (1995) "Many-body theory for semiconductor microcavity lasers", Phys. Rev., A52, $1712-1727$.

[25] DuBois, D. F. (1967). "Nonequilibrium statistical mechanics of plasmon and radiation", in Lectures in Theoretical Physics, New York: Gordon \& Breach, pp. $469-620$.

[26] Horie, C. and Krumhansl, J. A. (1964). "Boltzmann equation in a phonon system", Phys. Rev., A136, 1397 1407.

[27] Buot, F. A. (1972). "On the relaxation rate spectrum of phonons", J. Phys. C: Solid State Phys., 5, 5-14.

[28] Benin, D. (1975). "Phonon viscosity and wide-angle phonon scattering in superfluid helium", Phys. Rev., B11, $145-149$; H. Beck, "On the temperature behavior of second sound and Poiseuille flow", Z. Physik, B 20, 313322,1975 ; B. Perrin, "Sound propagation and vibrational relaxation in molecular crystals", J. Chemie Phys., 82, $191-197,1985$

[29] Agronovich, V. M. and Konobeev, Yu. V. (1964). "Theory of the dielectric permittivity of crystals", Sov. Phys.-Solid state, 5, 1858.

\section{Authors' Biography}

Dr. Felix A. Buot has served on the research staff of the University of London, ICTP (Trieste, Italy), McGill, St. Francis Xavier, Stanford, and Cornell University. He is a Fellow of the Washington Academy of Sciences, and President of the Philippine-American Academy of Science and Engineering. $\mathrm{He}$ is a member of the Editorial Board, Transport Theory and Statistical Physics. He was a UNDP Consultant, 1993 and 1996. His interests include device performance \& reliability, optoelectronics, nonequilibrium quantum theory, multiband dynamics, and physics of computation. $\mathrm{He}$ is a Research Physicist at the U.S. Naval Research Laboratory. 

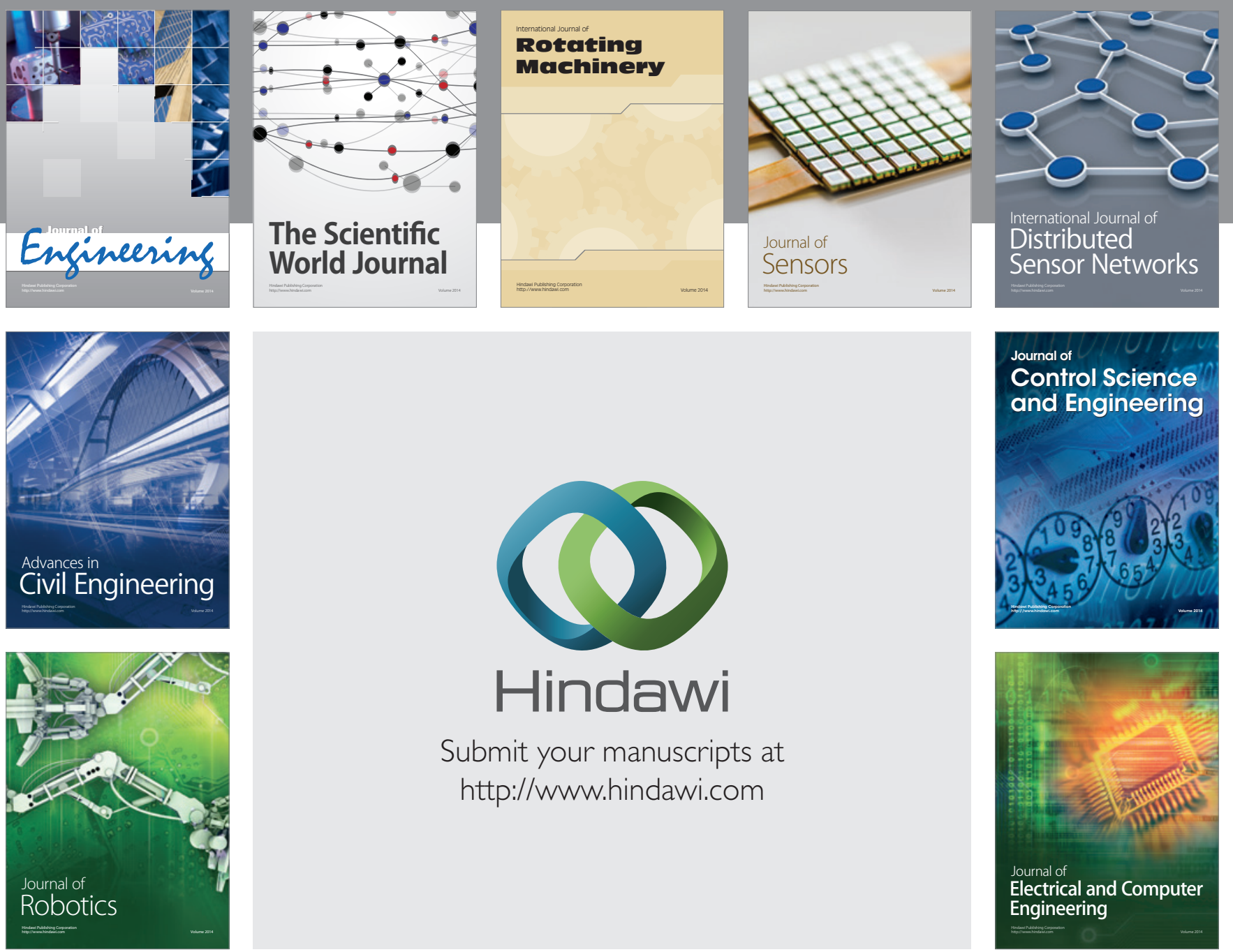

Submit your manuscripts at

http://www.hindawi.com
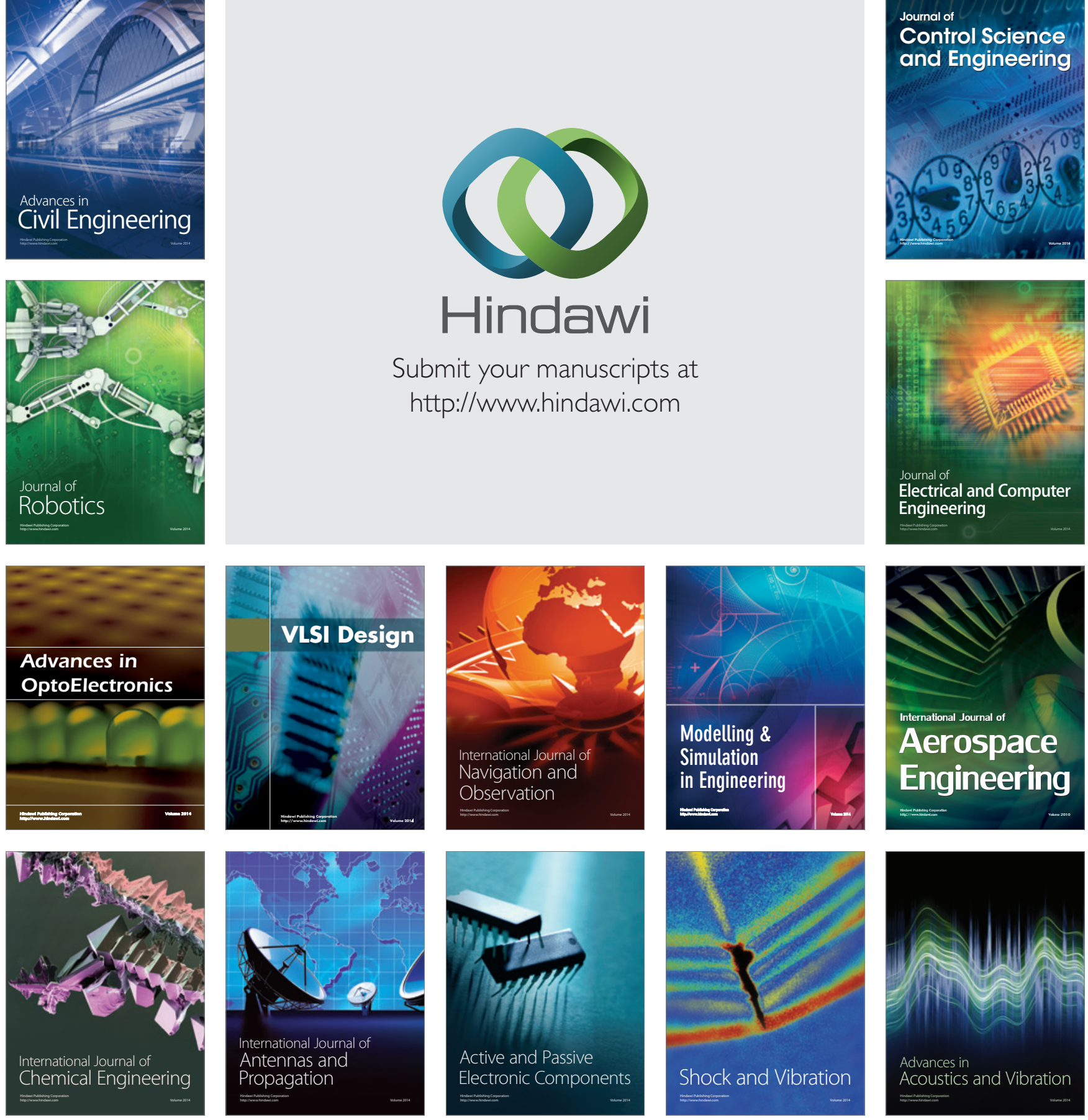